\title{
Particle shape dependence in 2D granular media
}

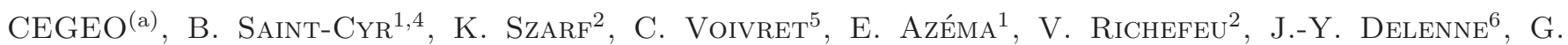

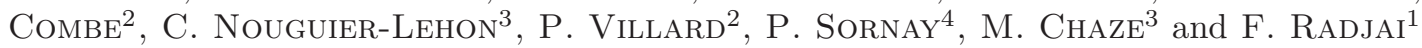

1 University Montpellier 2, CNRS, LMGC UMR 5508, Place Eugène Bataillon, F-34095 Montpellier Cedex, France.

2 UJF-Grenoble 1, Grenoble-INP, CNRS UMR 5521, 3SR Lab., B.P. 53, F-38041 Grenoble Cedex 09, France.

구 University of Lyon, Ecole Centrale de Lyon, LTDS UMR CNRS 5513, 36 avenue Guy de Colongue, F- 69134 Ecully cedex, France.

4 CEA, DEN, SPUA, LCU, F-13108 Saint Paul Lez Durance, France.

5 SNCF Innovation and Research Immeuble Lumière, 40 Avenue des Terroirs de France, F-75611 Paris cedex 12, of France.

G IATE, UMR 1208 INRA-CIRAD-Montpellier Supagro-UM2, 2 place Pierre Viala, F-34060 Montpellier cedex 01, France.

N'

PACS $45.70 .-\mathrm{n}$ - First pacs description
PACS $81.05 . \mathrm{Rm}$ - Second pacs description
PACS $61.43 . \mathrm{Hv}$ - Third pacs description

\begin{abstract}
Particle shape is a key to the space-filling and strength properties of granular matter. We consider a shape parameter $\eta$ describing the degree of distortion from a perfectly spherical shape. Encompassing most specific shape characteristics such as elongation, angularity and nonconvexity, $\eta$ is a low-order but generic parameter that we used in a numerical benchmark test for a systematic investigation of shape-dependence in sheared granular packings composed of particles of different shapes. We find that the shear strength is an increasing function of $\eta$ with nearly the same trend for all shapes, the differences appearing thus to be of second order compared to $\eta$. We also observe a nontrivial behavior of packing fraction which, for all our simulated shapes, increases with $\eta$ from the random close packing fraction for disks, reaches a peak considerably higher than that for disks, and subsequently declines as $\eta$ is further increased. These findings suggest that a low-order description of particle shape accounts for the principal trends of packing fraction and shear strength. Hence, the effect of second-order shape parameters may be investigated by considering different shapes at the same level of $\eta$.
\end{abstract}

The hard-sphere packing is at the heart of various models for the rheology and (thermo)dynamical properties of ạmorphous states of matter including liquids, glasses and granular materials 1,2. Such models reflect both the purely geometrical properties of sphere packings, e.g. the order-disorder transition with finite volume change 3 , and emergent properties arising from collective particle interactions, e.g. force chains and arching in static piles [4. As to non-spherical particle packings, rather recent results suggest that such packings exhibit higher shear strength than sphere packings [5-15, and may approach unusually high packing fractions [2,16 18. However, a systematic and quantitative investigation of shape-dependence is still largely elusive since particle shape characteristics such as

(a) Collaborative group "Changement d'Echelle dans les GEOmatériaux" (scale change in geomaterials) elongation, angularity, slenderness and nonconvexity are described by distinct groups of parameters, and the effect of each parameter is not easy to isolate experimentally.

In order to evaluate the shape-dependence of general granular properties such as packing fraction, shear strength and internal structure for particles of different shapes, we designed a numerical benchmark test that was simulated and analyzed by the members of a collaborative group (CEGEO). The idea of this test is that various non-spherical or non-circular shapes can be characterized by their degree of distortion from a perfectly spherical or circular shape. Let us consider an arbitrary 2D shape as sketched in Fig. 1. The border of the particle is fully enclosed between two concentric circles: a circumscribing circle of radius $R$ and an inscribed circle of radius $R-\Delta R$. We define the $\eta$-set as the set of all shapes with borders 


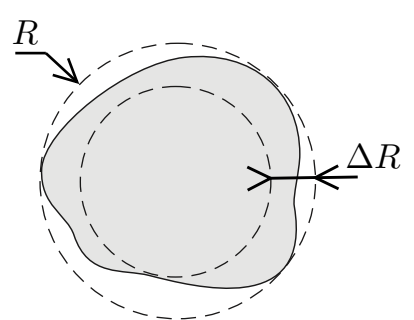

Fig. 1: An arbitrary particle shape represented by a concentric pair of circumscribing and inscribed circles.

enclosed between a pair of concentric circles (spheres in $3 \mathrm{D})$, touching both circles and having the same ratio

$$
\eta=\frac{\Delta R}{R}
$$

Four different particle shapes belonging to the same $\eta$ set are shown in Fig. 2, A non-zero value of $\eta$ corresponds to non-convexity for A-shape, elongation for B-shape, angularity for C-shape, and a combination of angularity and elongation for D-shape.
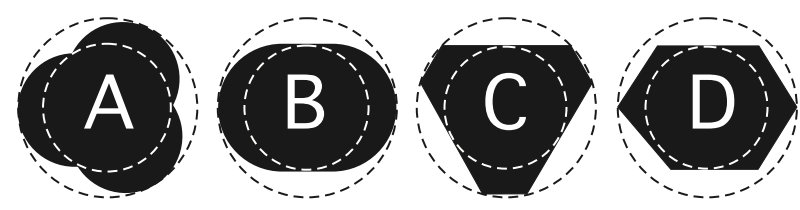

Fig. 2: Four different shapes belonging to the same $\eta$-set with $\eta=0.4$ : trimer (A), rounded-cap rectangle (B), truncated triangle $(\mathrm{C})$, and elongated hexagon (D).

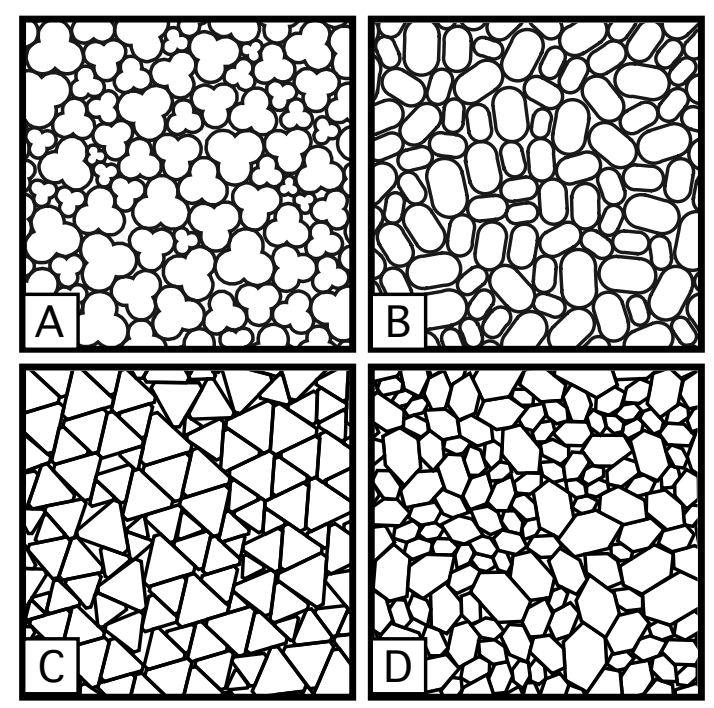

Fig. 3: Snapshots of the simulated packings in the densest isotropic state for $\eta=0.4$.

The parameter $\eta$ is obviously a rough low-order shape parameter; see also [19]. But, encompassing most specific shape parameters, it provides a general framework in

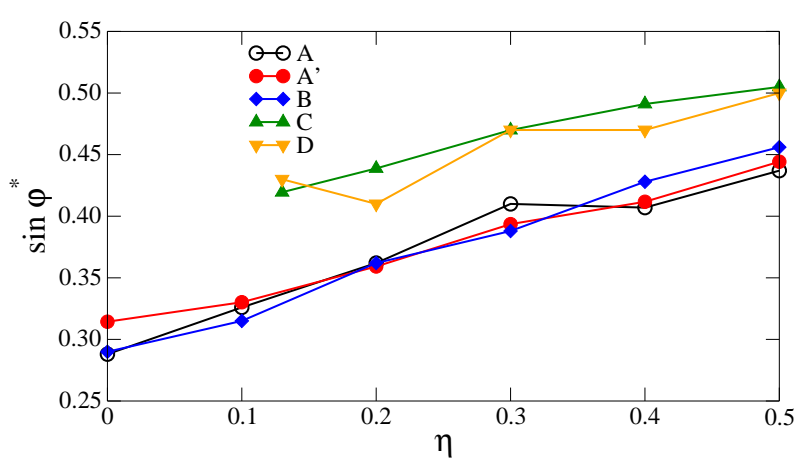

Fig. 4: Shear strength $\sin \varphi^{*}$ of packings composed of various particle shapes (see Fig. 2) as a function of $\eta$.

which shape-dependence may be analyzed among particles of very different shapes. Within an $\eta$-set, each specific shape may further be characterized by higher-order parameters. The issue that we address in this Letter is to what extent the packing fraction and shear strength are controlled by $\eta$ and in which respects the behavior depends on higher-order shape parameters

The benchmark test is based on the four shapes of Fig. 2. The A-shape (trimer) is composed of three overlapping disks touching the circumscribing circle and with their intersection points lying on the inscribed circle; the B-shape (rounded-cap rectangle) is a rectangle touching the inscribed circle and juxtaposed with two half-disks touching the circumscribing circle; the C-shape (truncated triangle) is a hexagon with three sides constrained to touch the inscribed circle and all corners on the circumscribing circle; and the D-shape (elongated hexagon) is an irregular hexagon with two sides constrained to touch the inscribed circle and two corners lie on the circumscribing circle. The range of geometrically defined values of $\eta$ for a given shape (defined by a construction method) has in general a lower bound $\eta_{0}$. For $\mathrm{A}$ and $\mathrm{B}$, the particle shape changes continuously from a disk, so that $\eta_{0}=0$ whereas we have $\eta_{0}=1-\sqrt{3} / 2 \simeq 0.13$ for $\mathrm{C}$ and $\mathrm{D}$.

Two different discrete element methods (DEM) were used for the simulations: contact dynamics (CD) and molecular dynamics (MD). In the CD method, the particles are treated as perfectly rigid [20] whereas a linear spring-dashpot model was used in MD simulations with stiff particles $\left(k_{n} / p_{0}>10^{3}\right.$, where $k_{n}$ is the normal stiffness and $p_{0}$ refers to the confining pressure) [21. The trimers were simulated by both methods for all values of $\eta$. We refer below as A (for CD) and A' (for MD) to these simulations. The packing $\mathrm{C}$ was simulated by $\mathrm{MD}$ whereas the packings $\mathrm{B}$ and $\mathrm{D}$ were simulated by CD. In CD simulations, the coefficient of restitution was set to zero. In MD simulations, the damping parameter was taken very close to the critical damping coefficient so that the restitution coefficient was also negligibly small 22. Note that in quasi-static flow, the relaxation time of the particles is short enough (compared to the inverse shear rate) to al- 


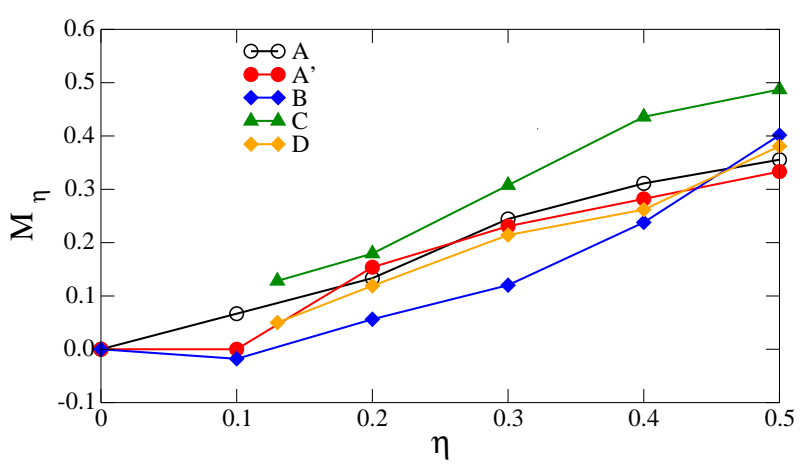

Fig. 5: Friction mobilization in the steady state as a function of $\eta$ for different particle shapes.

low for efficient dissipation of kinetic energy in each time step. For this reason, in contrast to granular gases, the exact values of the damping parameters or restitution coefficients have practically no influence on the numerical data analyzed below 23 .

For each shape, several packings of 5000 particles were prepared with $\eta$ varying from 0 to 0.5 . To avoid longrange ordering, a size polydispersity was introduced by taking $R$ in the range $\left[R_{\min }, R_{\max }\right]$ with $R_{\max }=3 R_{\min }$ and a uniform distribution of particle volumes. A dense packing composed of disks $(\eta=0)$ was first constructed by means of random deposition in a box 24. For other values of $\eta$, the same packing was used with each disk serving as the circumscribing circle. The particle was inscribed with the desired value of $\eta$ and random orientation inside the disk. This geometrical step was followed by isotropic compaction of the packings inside a rectangular frame. The gravity $g$ and friction coefficients between particles and with the walls were set to 0 during compaction in order to avoid force gradients. Fig. 3 displays snapshots of the packings for $\eta=0.4$ at the end of isotropic compaction 1 .

The isotropic samples were sheared by applying a slow downward velocity on the top wall with a constant confining stress acting on the lateral walls. During shear, the friction coefficient $\mu$ between particles was set to 0.5 and to 0 with the walls. The shear strength is characterized by the internal angle of friction $\varphi$ defined by

$$
\sin \varphi=\frac{\sigma_{1}-\sigma_{2}}{\sigma_{1}+\sigma_{2}}
$$

where the subscripts 1 and 2 refer to the principal stresses. $\sin \varphi$ increases rapidly from zero to a peak value before relaxing to a constant material-dependent value $\sin \varphi^{*}$, which defines the shear strength at large strain at a steady stress state.

Figure 4 shows the dependence of $\sin \varphi^{*}$ with respect to $\eta$ for our different shapes. Remarkably, $\sin \varphi^{*}$ increases with $\eta$ at the same rate for all shapes. The data nearly coincide between the $\mathrm{A}$ and $\mathrm{B}$ shapes, on the one hand, and

\footnotetext{
${ }^{1}$ Animation videos of the simulations can be found at www.cgpgateway.org/ref012.
}

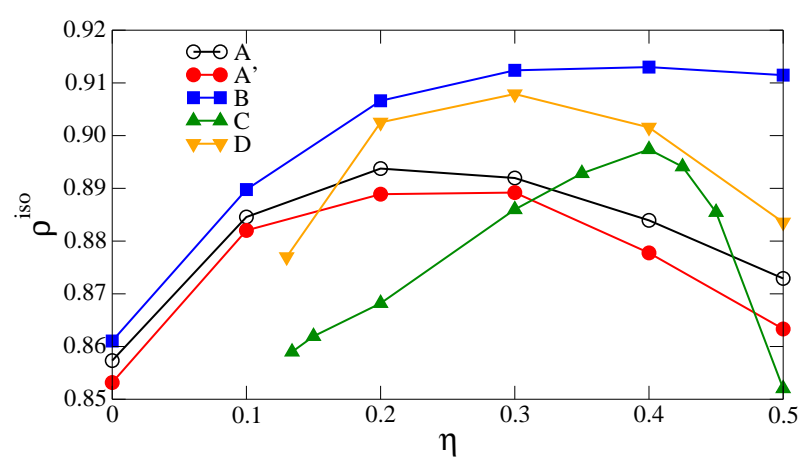

Fig. 6: Packing fraction in the isotropic state as a function of $\eta$ for different particle shapes.

between $\mathrm{C}$ and $\mathrm{D}$ shapes, on the other hand. This suggests that nonconvex trimers and rounded-cap rectangles, in spite of their very different shapes, belong to the same family (rounded shapes). In the same way, the truncated triangles and elongated hexagons seem to belong to the family of angular particles and exhibit a shear strength slightly above that of rounded shapes. Note also that the results are robust with respect to the numerical approach as the packings $\mathrm{A}$ and $\mathrm{A}^{\prime}$ were simulated by two different methods.

The increase of shear strength with $\eta$ may be attributed to the increasing frustration of particle rotations as the shape deviates from a disk [11,25]. Since the particles may interact at two or three contact points (A-shape) or through side-to-side contacts (shapes B, C and D), the kinematic constraints increase with $\eta$ and frustrate the particle displacements by rolling. The restriction of rolling leads to enhanced role of friction in the mechanical equilibrium and relative sliding of particles during deformation. A related static quantity is the mean friction mobilization defined by $M=\left\langle f_{t} /\left(\mu f_{n}\right)\right\rangle$, where $f_{t}$ is the magnitude of the friction force, $f_{n}$ is the normal force, and the average is taken over all force-bearing contacts in the system.

To evaluate the effect of particle shape, we consider the parameter

$$
M_{\eta}=\frac{M(\eta)}{M(\eta=0)}-1
$$

as a function of $\eta$ for different shapes, where $M(\eta=0)$ is the friction mobilization for circular particles. Fig. 5 shows that $M_{\eta}$ is a globally increasing function of $\eta$ for all shapes. The parameter $\eta$ appears also in this respect to account for the global trend of friction mobilization, and the differences observed in Fig. 5 among different shapes are rather of second order.

We also observe that the proportions of double and triple contacts for A-shape packings and the proportion of side-to-side contacts for other shapes increase with $\eta$. For noncircular particles, one should distinguish the coordination number $Z$, defined as the mean number of contacting neighbors per particle, from the "contact coordination number" $Z_{c}$ defined as the mean number of contacts per 


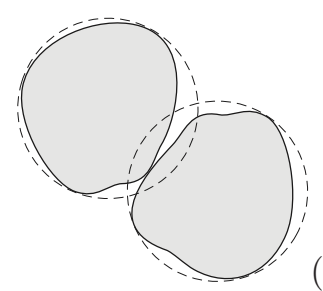

(a)

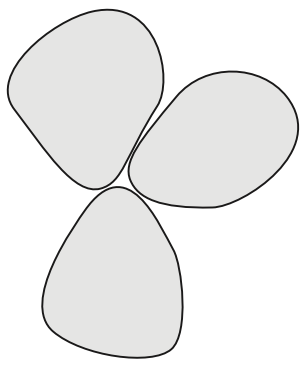

(b)

Fig. 7: Pore volume reduction by (a) overlap between selfporosities; (b) steric pores.

particle. Obviously, for the calculation of both $Z$ and $Z_{c}$ only the force-bearing contacts and non-floating particles are taken into account 26. We have $Z=Z_{c} \simeq 4$ for the disks in the initial state prepared with $\mu=0$. This value corresponds to an isostatic state in which one expects $Z=2 N_{f}$, where $N_{f}$ is the number of degrees of freedom of a particle 27. For frictionless disks, we have $N_{f}=2$ (two translational degrees of freedom), leading to $Z=4$. For noncircular shapes, we have $N_{f}=3$ since the rotational degrees of freedom take part in the mechanical equilibrium of the particles. Hence, if isostaticity holds also for noncircular frictionless particles, we expect $Z=6$. We observe instead $Z<5$ for all our packings. However, we find $Z_{c} \simeq 6$ for $\eta \neq 0$ if each side-to-side contact is counted twice, representing two independent constraints. This result is consistent with the isostatic nature of a packing of frictionless noncircular particles and shows that the packings of noncircular shape are not under-constrained as previously suggested 28 . For $\mu=0.5$, the packings are no more isostatic and $Z$ and $Z_{c}$ vary only slightly with $\eta$ with values in the range 3 to 4 for $Z$ and in the range 4 to 5 for $Z_{c}$ in the course of shearing.

We now focus on the packing fraction which crucially depends on particle shape. Fig. [6] shows the packing fraction $\rho^{i s o}$ in the initial isotropic state as a function of $\eta$. We observe a nontrivial behavior for all particle shapes: the packing fraction increases with $\eta$, passes by a peak depending on each specific shape and subsequently declines. For the B-shape a sharp decrease of $\rho^{i s o}$ occurs beyond $\eta=0.5$ as was shown in 10 .

This unmonotonic behavior of packing fraction was observed by experiments and numerical simulations for spheroids as a function of their aspect ratio [2, 16, 17, 2830. The decrease of the packing fraction is attributed to the excluded-volume effect that prevails at large aspect ratios and leads to increasingly larger pores which cannot be filled by the particles 29]. The observation of this unmonotonic behavior as a function of $\eta$ for different shapes indicates that it is a generic property depending only on deviation from circular shape. This behavior may thus be explained from general considerations involving the parameter $\eta$ but with variations depending on second-order shape characteristics.

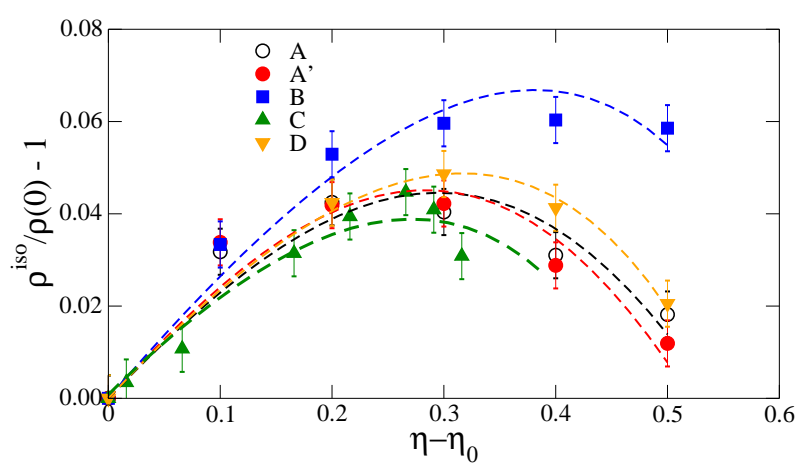

Fig. 8: Normalized packing fractions fitted by Eq. (6).

A plausible second-order parameter is

$$
\nu=\frac{V_{p}}{\pi R^{2}}
$$

where $V_{p}$ is the particle volume in $2 \mathrm{D}$. Its complement $1-\nu$ is the "self-porosity" of a particle, i.e. the unfilled volume fraction inside the circumscribing circle. Keeping the radius $R$ of the circumscribing circle constant, $\rho^{i s o}=$ $V_{p} / V$ varies with $\eta$ as a result of the relative changes of $V_{p}$ and the mean volume $V$ per particle. The free (pore) volume per particle is $V_{f}=V-V_{p}$.

At $\eta=0$, the free volume $V_{f}$ is only composed of steric voids, i.e. voids between three or more particles, and the packing fraction is given by $\rho(0)=\pi R^{2} / V(0)$. For $\eta>0$, the void patterns are more complex but can be described by considering the generic shape of particles belonging to a given $\eta$-set. The borders of a particle involve "hills", which are the parts touching the circumscribing circle, and "valleys" touching the inscribed circle. The volume $V$ per particle varies with $\eta$ by two mechanisms. First, the hills of a particle may partially fill the valleys of a neighboring particle; Fig. 7(a). Secondly, the steric voids between the hills shrink as $\eta$ increases due to the increasing local curvature of the touching particles; Fig. 7(b). To represent this excess or loss of pore volume due to the specific jamming configurations induced by particle shapes, we introduce the function $h(\eta)$ by setting

$$
V(\eta)=V\left(\eta_{0}\right)-\pi R^{2} h(\eta)
$$

with $h\left(\eta_{0}\right)=0$. With these assumptions, the packing fraction is expressed as

$$
\rho(\eta)=\frac{\nu(\eta) \rho\left(\eta_{0}\right)}{1-h(\eta) \rho\left(\eta_{0}\right)} .
$$

The function $\nu(\eta)$ is known for each shape but $h(\eta)$ needs to be estimated. A second-order polynomial approximation

$$
h(\eta)=\alpha\left(\eta-\eta_{0}\right)+\beta\left(\eta-\eta_{0}\right)^{2}
$$

together with Eq. 6 allows us to recover the correct trend and to fit the data as shown in Fig. 8 The error bars 
represent the variability at $\eta_{0}$ assumed to be the same for all other values of $\eta$. The parameter $\alpha$ ensures the increase of packing fraction with $\eta$ at low values of the latter and it basically reflects the shrinkage of steric pores (Fig. 7(b)) whereas $\beta$ accounts for the overlap between circumscribing circles (Fig. 7(a))) and is responsible for the subsequent decrease of the packing fraction.

The fitting parameters in Fig. 8 are $\alpha \simeq 1.30,1.29$, $1.14,1.17$ and $\beta \simeq 1.23,1.20,0.23,0.20$ for $\mathrm{C}, \mathrm{A}, \mathrm{D}$ and $\mathrm{B}$ shapes, respectively with increasing peak value. Note that the values of $\beta$ are considerably smaller for B and D that have an elongated aspect and for which the overlapping of self-porosities prevails as compared to A and C for which the shrinkage of the initial pores is more important.

In summary, our benchmark simulations show that a low-order shape parameter $\eta$, describing deviation with respect to circular shape, controls to a large extent both the shear strength and packing fraction of granular media composed of noncircular particles in 2D. The shear strength is roughly linear in $\eta$ whereas the packing fraction is unmonotonic. Our simple model for this unmonotonic behavior is consistent with the numerical data for all shapes. It is governed by a first-order term in $\eta$ for the shrinkage of the initial steric pores and a second-order term in $\eta$ for the creation of large pores by shape-induced steric pores. The effect of higher-order shape parameters may be analyzed also in this framework in terms of differences in packing fraction and shear strength among various shapes belonging to the same $\eta$-set. An interesting issue to be addressed in future is whether a generic second-order parameter accounting for such differences exists. Another aspect that merits further investigation is the joint effects of size polydispersity and particle shape. The shear strength is independent of particle size polydispersity as a result of the capture of force chains by the class of larger particles 31. But the packing fraction and force and contact anisotropy depend on both shape and polydispersity.

\section{$* * *$}

We thank B. Cambou and F. Nicot for stimulating discussions. We also acknowledge financial support of the French government through the program PPF CEGEO.

\section{REFERENCES}

[1] Binder K. and Ков W., Glassy materials and disordered solids (World Scientific) 2005.

[2] Man W., Donev A., Stillinger F., Sullivan M., Russel W., Heeger D., S.Inati, Torquato S. and Chaikin P., Phys. Rev. Lett., 94 (2005) 198001.

[3] Torquato S., Truskett T. M. and Debenedetti P. G., Phys. Rev. Lett., 84 (2000) 2064.

[4] Radjai F., Wolf D. E., Jean M. and Moreau J., Phys. Rev. Letter, 80 (1998) 61.

[5] Oundfel H. and Rothenburg L., Mechanics of Materials, 33 (2001) 201.
[6] Mirghasemi A., Rothenburg L. and Maryas E., Geotechnique, 52 (2002) N 3, 209.

[7] Nouguier-Lehon C., Cambou B. and Vincens E., Int. J. Numer. Anal. Meth. Geomech., 27 (2003) 1207.

[8] Azéma E., Radjai F., Peyroux R. and Saussine G., Phys. Rev. E, 76 (2007) 011301.

[9] Azéma E., Radjai F. and Saussine G., Mechanics of Materials, 41 (2009) 721.

[10] Azéma E. and Radjai F., Phys. Rev. E, 81 (2010) 051304.

[11] Estrada N., Azéma E., Radjai F. and Taboada A., Phys. Rev. E, 84 (2011) 011306.

[12] Azéma E. and Radjai F., Phys. Rev. E, 85 (2012) 031303.

[13] Nouguier-Lehon C., Comptes Rendus Mécanique, 338 (2010) 587.

[14] Saint-Cyr B., Delenne J.-Y., Voivret C., Radjai F. and Sornay P., Phys. Rev. E, 84 (2011) 041302.

[15] Szarf K., Combe G. and Villard P., Powder Technology, 208 (2011) 279.

[16] Donev A., Stillinger F., Chaikin P. and Torquato S., Phys. Rev. Lett., 92 (2004) 255506.

[17] Donev A., Cisse I., Sachs D., Variano E., Stillinger F., Connelly R., Torquato S. and Chaikin P., Science, 303 (2004) 990.

[18] Jiao Y., Stillinger F. H. and Torquato S., Phys. Rev. E, 041304 (2010) 1.

[19] Pöschel T. and Buchholtz V., Phys. Rev. Lett., 71 (1993) 3963.

[20] Radjai F. and Richefeu V., Mechanics of Materials, 41 (2009) 715.

[21] Combe G. and Roux J.-N., Discrete numerical simulation, quasistatic deformation and the origins of strain in granular materials in proc. of Deformation Characteristics of Geomaterials, edited by ET AL. D. B., 2003 pp. 1071-1078.

[22] Brilliantov N. V., Spahn F., Hertzsch J.-M. and Pöschel T., Phys. Rev. E, 53 (1996) 5382.

[23] Roux J.-N. and Chevolr F., Dimensional analysis and control parameter in book. of Discrete-element Modeling of Granular Materials (ISTE-Wiley), edited by F.RADJAI AND F.Dubois., 2011 Ch. 8 pp. 223-253.

[24] Voivret C., Radjai F., Delenne J.-Y. and Youssoufi M. S. E., Phys. Rev. E, 76 (2007) 021301.

[25] Estrada N., Taboada A. and Radjai F., Phys. Rev. E, 78 (2008) 021301.

[26] Combe G. and Roux J.-N., Construction of granular assemblies under static loading in book. of ,Discrete-element Modeling of Granular Materials (ISTE-Wiley), edited by F.RAdjai And F.Dubois., 2011 Ch. 6 pp. 153-180.

[27] Agnolin I. and Roux J.-N., Phys; Rev. E, 76 (2007) 061302.

[28] Donev A., Connelly R., Stillinger F. and Torquato S., Phys. Rev. E, 75 (2007) 051304.

[29] Williams S. and Philipse A., Phys. Rev. E, 67 (2003) 051301.

[30] Sacanna S., Rossi L., Wouterse A. and Philipse A., Journal of Physics, 19 (2007) 376108.

[31] Voivret C., Radjai F., Delenne J.-Y. and El YousSOufi M. S., Phys. Rev. Lett., 102 (2009) 178001. 